\title{
Lunar albedo at hydrogen Lyman $\alpha$ by the NOZOMI/UVS
}

\author{
M. Taguchi ${ }^{1}$, G. Funabashi ${ }^{2}$, S. Watanabe ${ }^{3}$, Y. Takahashi ${ }^{2}$, and H. Fukunishi ${ }^{2}$ \\ ${ }^{1}$ National Institute of Polar Research, Tokyo 173-8515, Japan \\ ${ }^{2}$ Department of Geophysics, Tohoku University, Sendai 980-8578, Japan \\ ${ }^{3}$ Department of Earth and Planetary Science, Hokkaido University, Sapporo 060-0810, Japan
}

(Received July 14, 2000; Revised September 11, 2000; Accepted September 20, 2000)

\begin{abstract}
The geometric albedo of the Moon at the wavelength of hydrogen Lyman $\alpha$ (HLy $\alpha 121.6 \mathrm{~nm}$ ) was derived from an imaging observation by the ultraviolet imaging spectrometer (UVS) during the lunar encounter of the NOZOMI spacecraft. The solar HLy $\alpha$ irradiance data measured by the UARS/SOLSTICE at the time of UVS observation were adopted. We obtained an average geometric albedo of $5.2 \pm 0.9 \%$ for the sunlit region where the UVS observed. Our result agrees with the geometric albedos obtained from the observations by Apollo 17 and Astro-2/HUT, though the observation geometry and area are completely different from each other. There exists a significant difference of the albedos from place to place in the observed lunar surface ranging from $2.3 \pm 1.1 \%$ to $6.0 \pm 1.0 \%$. It is noted that the observed contrast at the FUV wavelength is positive to that seen in the visible region.
\end{abstract}

\section{Introduction}

Reflectivity of a planetary disk is one of the most primitive parameters obtained by optical remote sensing from a distance. There are two definitions of planetary reflectance or albedos: the Bond albedo and geometric albedo. The former is defined by the ratio of the total amount of reflected light to the total amount of incident light. This value is important especially for planets with atmosphere in order to estimate total energy input. The latter is defined by the ratio of the actual intensity of light reflected by a planetary disk to that of a Lambertian surface or a perfect diffuser with the same size. The geometric albedo can be calculated not only for an entire planetary disk but also for a small part of a disk. The geometric albedo includes information on surface material and condition of planets without atmosphere.

The geometric albedo of the lunar surface in the FUV region was firstly reported by Lucke et al. (1976) using the data obtained by a scanning far-ultraviolet spectrometer onboard Apollo 17. From the observation data taken while the orbiter was in a lunar orbit for five days, geometric albedos of $4.4 \%-$ $6.3 \%$ in the wavelength region of $122-168 \mathrm{~nm}$ were obtained. The second measurement was made by an ultraviolet spectrometer during fly-by of Mariner 10 (Wu and Broadfoot, 1977). The result shows rather small albedos compared with those obtained by Apollo 17. Another observation, though the spectral data did not include hydrogen Lyman $\alpha$ (HLy $\alpha$ $121.6 \mathrm{~nm}$ ), was made by the Hopkins Ultraviolet Telescope onboard the Astro-2 Space Shuttle mission (hereafter Astro2/HUT) obtaining the geometric albedos of $3.7 \%$ to $4.1 \%$ in the spectral region from $183 \mathrm{~nm}$ to $125 \mathrm{~nm}$ (Henry et al., 1995). This result is more accurate than the past two observations and shows rather small spectral dependence of the

Copy right (C) The Society of Geomagnetism and Earth, Planetary and Space Sciences (SGEPSS); The Seismological Society of Japan; The Volcanological Society of Japan; The Geodetic Society of Japan; The Japanese Society for Planetary Sciences. lunar albedo.

The NOZOMI spacecraft is the first Japanese Mars orbiter launched on July 3, 1998. It spent about a half year in an orbit around the Earth before it was inserted into a Mars transfer orbit. During the Earth orbit it encountered with the Moon twice for swing-by. An ultraviolet imaging spectrometer (UVS) observed the lunar surface at the two lunar encounters. The geometric albedo of the Moon at the wavelength of HLy $\alpha$ was derived from the spectral data obtained at the first lunar encounter. Unfortunately, the distance between the spacecraft and the Moon during the observation period at the second encounter was too large to obtain useful data of the lunar surface.

\section{Observation}

Details of the UVS instrument onboard the NOZOMI spacecraft are described in Taguchi et al. (2000). The UVS$\mathrm{G}$ consists of a collecting parabolic mirror, a slit, a concave grating, and photo-detectors and measures the spectral region of $110-310 \mathrm{~nm}$ with a spectral resolution of $2-3 \mathrm{~nm}$. A spectrum in the FUV region is detected by an micro-channel plate with strip anodes. The UVS takes an image using spin and orbital motions of the spacecraft.

Observation of the lunar surface was made while the NOZOMI spacecraft approached the Moon on September 24, 1998. A lunar image at the wavelength of HLy $\alpha$ is shown in Plate 1. Figure 1 illustrates schematic geometry of the UVS observation. The distance between the center of the Moon and the spacecraft varied from 9,240 km at 05:44 UT to $6,800 \mathrm{~km}$ at $06: 33 \mathrm{UT}$. The change in the distance causes distortion of the lunar outline depicted in the image. For comparison a visible image of the Moon taken by the Mars Imaging Camera (MIC) onboard the NOZOMI spacecraft (see http://www.planet-b.isas.ac.jp/MIC/ MIC-e.html) is shown in Plate 2. This image is com- 


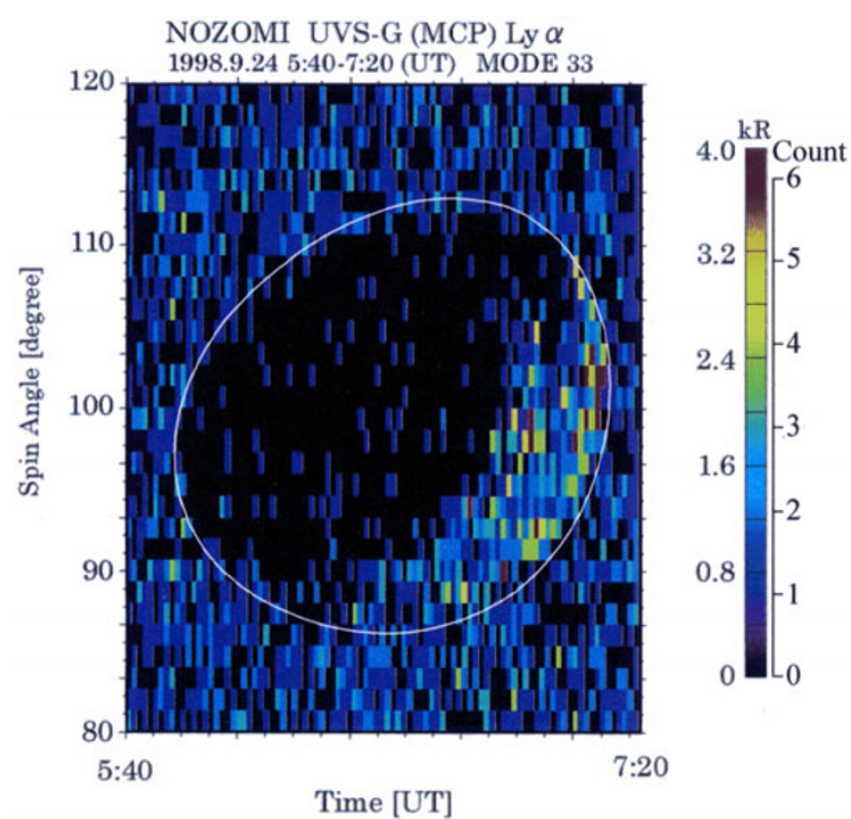

Plate 1. A HLy $\alpha$ image of the Moon taken by the UVS-G at the first encounter of the NOZOMI spacecraft to the Moon on September 24, 1998. The integration time for each pixel was $0.1 \mathrm{sec}$. A white oval shows the approximate outline of the Moon.

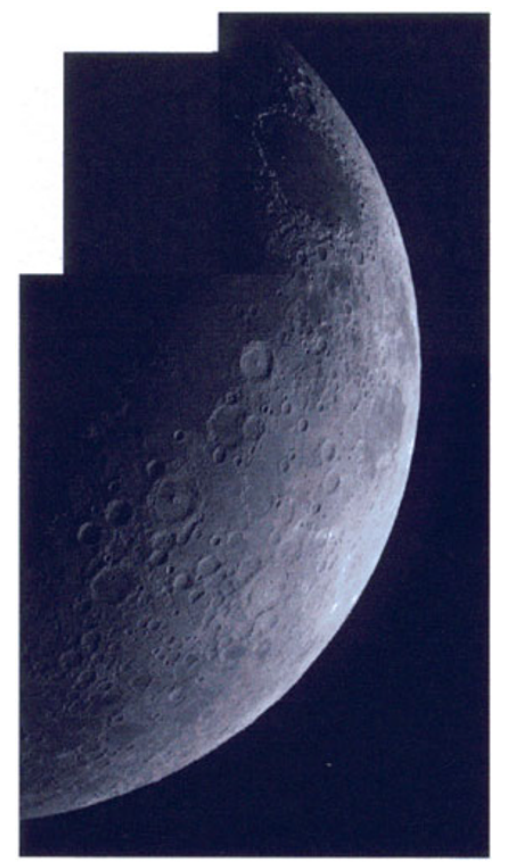

Plate 2. A composite image of the Moon taken by a visible camera (MIC) onboard NOZOMI on September 24, 1998. Sea of Crises is seen at the upper side.

position of several shots taken during the approach to the Moon.

Notation of the angles relevant to reflection of solar light at the lunar surface is also shown in Fig. 1. The angle between the direction of light incident into the lunar surface and the direction of observation $\alpha$ was almost constant around $130^{\circ}$ during the observation period. The angle between the surface normal and the direction of incident light $i$ varied from $52^{\circ}$

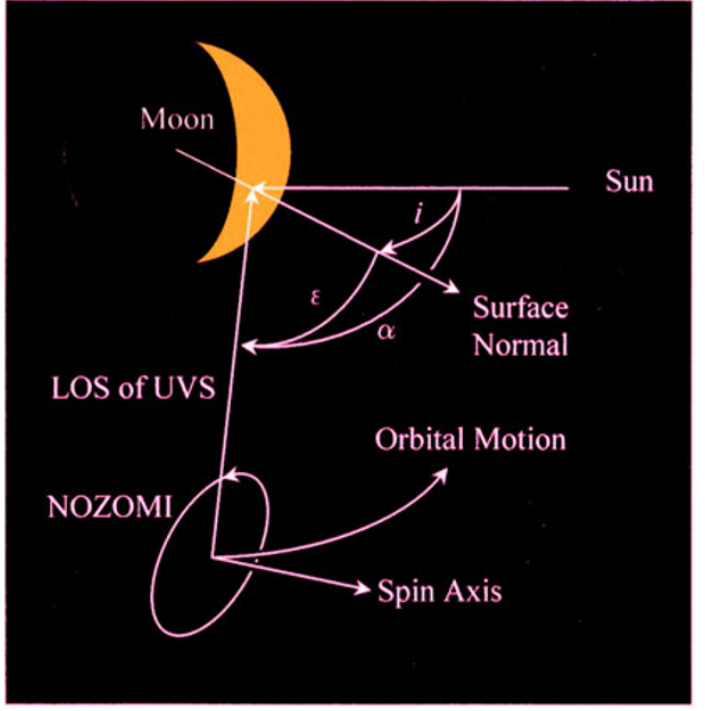

Fig. 1. Schematic of the observation geometry and notation of the angles relevant to the UVS observation of the lunar surface.

to $90^{\circ}$, while the angle between the surface normal and the direction of observation $\epsilon$ varied from $43^{\circ}$ to $90^{\circ}$.

\section{Data Analysis}

The derivation of the geometric albedo is described in Hapke (1963). Here we follow Lucke et al. (1976) for notation of the equations. The observed intensity $I(i, \epsilon, \alpha)$ is expressed as

$$
I(i, \epsilon, \alpha)=I_{0} A \Omega b(\Sigma(\alpha) B(\alpha, g)) /(1+\cos \epsilon / \cos i),
$$

where $I_{0}$ is intensity of incident radiation, $A \Omega$ instrumental throughput, $b$ the total average reflectivity of the individual objects, $\Sigma(\alpha)$ average scattering law of the individual objects, and $B(\alpha, g)$ the retrodirective function. The assumptions on which Eq. (1) is derived are as follows (Hapke, 1963): 1) The surface consists of a semi-infinite layer of objects large compared with a wavelength of light and arranged in an open network into which light from any direction can penetrate freely. The objects are located irregularly enough within this structure so that on a macroscopic scale the medium appears isotropic and homogeneous. 2) The reflectivity of the individual objects is low. 3) The reflecting objects are oriented at random. 4) The intensity of light is exponentially attenuated in proportion to the path length of the ray through the medium. 5) All solid angles involved are small.

The functions $\Sigma(\alpha)$ and $B(\alpha, g)$ and a compaction parameter $g$ in Lucke et al. (1976) are adopted. The values of $i, \epsilon$, and $\alpha$ are calculated from the known positions of the Sun, the Moon and the spacecraft at the time of observation. Once we know the value of $I_{0}$ as described later, we can derive a value of $b$ from Eq. (1) and the geometric albedo $p$ according to Eq. (9) in Lucke et al. (1976):

$$
p=\frac{\pi I(i, \epsilon, 0)}{I_{0} A \Omega}=\frac{5 b}{6} .
$$

Surface luminosity data of the sunlit region where the fieldof-view of UVS swept are used to derive an average geomet- 
ric albedo. The data close to the limb, $\epsilon>72^{\circ}$, are excluded, because a part of the field-of-view may have looked at the space. The data close to the terminator, where $i>85^{\circ}$, are also excluded because of the shadowing problem. Geometric albedos are calculated using the spectral data only at HLy $\alpha$, though the UVS covers the spectral region from 110 through $310 \mathrm{~nm}$. The reasons why only this wavelength was chosen are that 1) the total counts of the UVS-G at HLy $\alpha$ are high enough compared with the other wavelengths and 2) the spectral response of the UVS-G was calibrated at three wavelengths including this wavelength (Taguchi et al., 2000).

We have to know the solar HLy $\alpha$ irradiance $I_{0}$ at the time of observation to deduce the geometric albedo. Since the NOZOMI spacecraft has no instrument to monitor the solar ultraviolet irradiance, the UARS/SOLSTICE data are adopted. The SOLSTICE measures the solar ultraviolet irradiance in the wavelength range from 119 to $420 \mathrm{~nm}$ with spectral resolution of 0.1 to $0.3 \mathrm{~nm}$, and the overall accuracy of absolute irradiance at the wavelength of HLy $\alpha$ is better than $10 \%(2 \sigma)$ (Woods et al., 1996). Daily mean solar spectral irradiance values are available from their WWW. At the time of UVS observation the sun was in the quiet period and no particular solar event was reported. Daily values of the solar irradiance on consecutive nine days including September 24,1998 show very slight variation of $\pm 1 \%$. Therefore, we consider that the daily mean value can be represented as the value at the time of UVS observation.

\section{Result and Discussion}

The result is shown in Table 1 together with the observations of lunar albedo at HLy $\alpha$ in the past. The error of $\pm 0.9 \%$ in our result includes not only statistical error in the measurement but also spatial variability of the albedo. Unfortunately the most recent and precise measurement by the Astro-2/HUT experiment derives albedos only at wavelengths longer than $125 \mathrm{~nm}$. However, the albedo in the wavelength range of 125-129 nm from Astro-2/HUT is added in Table 1 for comparison, because their results show very slight dependence on wavelength. The albedo derived from the Mariner 10 fly-by shows a significantly smaller value than the others. This value is also much smaller than the albedos at nearby wavelengths obtained from the same experiment $(\mathrm{Wu}$ and Broadfoot, 1977). Therefore, this measurement might contain an unknown error.

The Astro-2/HUT observations were conducted in nearly full-moon conditions, and, therefore, $\alpha=0^{\circ}$ and $i=\epsilon$. In such a geometry the backscattered component is significant in the reflected light, and the observed albedo is an average

Table 1. Measurements of lunar geometric albedos at HLy $\alpha$ and nearby wavelengths.

\begin{tabular}{ccc}
\hline Experiment & $\lambda(\mathrm{nm})$ & $p$ \\
\hline NOZOMI/UVS & 121.6 & $5.2 \pm 0.9 \%$ \\
APOLLO 17 & 121.6 & $6.3 \%$ \\
MARINER 10 & 121.6 & $2.1 \%$ \\
Astro-2/HUT & $125-129$ & $4.1 \%$ \\
\hline
\end{tabular}

over a hemisphere facing to the Earth. The spectrometer onboard Apollo 17 always looked at $30^{\circ}$ from the local vertical $\left(\epsilon=30^{\circ}\right)$ but $i$ varied from $5^{\circ}$ to $75^{\circ}$ and $\alpha$ from $10^{\circ}$ to $100^{\circ}$. The observation by Apollo 17 was limited to the particular area in the low latitudes. The geometry and area of the NOZOMI/UVS observation differ from those of Apollo 17 and Astro-2/HUT. Nevertheless our result generally agrees with those of Apollo 17 and Astro-2/HUT.

It is noted that there exists a significant difference of the albedos from place to place ranging from $2.3 \pm 1.1 \%$ to $6.0 \pm 1.0 \%$. As expected by comparing the HLy $\alpha$ and visible images shown in Plates 1 and 2 the observed contrast at HLy $\alpha$ exhibits same tendency as seen in the visible light. Contrast of the FUV images taken by the UVS in the other wavelengths (an example is shown in Taguchi et al. (2000)), though they are not so good in quality as the HLy $\alpha$ image, also resembles that of the HLy $\alpha$ image. This evidence suggests that the contrast reversal on the lunar surface occurs shorter than the wavelength of HLy $\alpha$. This is consistent with the observational result by Flynn et al. (1998), in which they found a high degree of reverse contrast at $20 \mathrm{~nm}$, whereas the $60 \mathrm{~nm}$ image shows no detectable contrast.

\section{Summary}

The geometric albedo of the Moon at the wavelength of HLy $\alpha$ was derived to be $5.2 \pm 0.9 \%$ for average of the sunlit region where the UVS observed. Our result is consistent with the geometric albedos obtained from the past observations by Apollo 17 and Astro-2/HUT. It is noted that there exists a spatial difference of the albedos ranging from $2.3 \pm 1.1 \%$ to $6.0 \pm 1.0 \%$, and the observed contrast is positive to that seen in the visible region.

Acknowledgments. The authors thank all the members of the NOZOMI project team for their effort in development, launch and operation of the NOZOMI spacecraft, and especially T. Mukai who kindly provided a lunar image taken by MIC. Valuable comments by M. Nakamura and T. Hiroi are appreciated.

\section{References}

Flynn, B. C., J. V. Vallerga, G. R. Gladstone, and J. Edelstein, Lunar reflectivity from Extreme Ultraviolet Explorer imaging and spectroscopy of the full Moon, Geophys. Res. Lett., 25, 3253-3256, 1998.

Hapke, B. W., A theoretical photometric function for the lunar surface, $J$. Geophys. Res., 68, 4571-4586, 1963.

Henry, R. C., P. D. Feldman, J. W. Kruk, A. F. Davidsen, and S. T. Durrance, Ultraviolet albedo of the Moon with the Hopkins Ultraviolet Telescope, Astrophys. J., 454, L69-L72, 1995.

Lucke, R. L., R. C. Henry, and W. G. Fastie, Far-ultraviolet albedo of the Moon, Astron. J., 81, 1162-1169, 1976.

Taguchi, M., H. Fukunishi, S. Watanabe, S. Okano, Y. Takahashi, and T. D. Kawahara, Ultraviolet imaging spectrometer (UVS) experiment on board the NOZOMI spacecraft: Instrumentation and initial results, Earth Planets Space, 52, 49-60, 2000.

Woods, T. N., D. K. Prinz, G. J. Rottman, J. London, P. C. Crane, R. P. Cebula, E. Hilsenrath, G. E. Brueckner, M. D. Andrews, O. R. White, M E. VanHoosier, L. E. Floyd, L. C. Herring, B. G. Knapp, C. K. Pankratz, and P. A. Reiser, Validation of the UARS solar ultraviolet irradiances: Comparison with the ATLAS 1 and 2 measurements, J. Geophys. Res., 101, 9541-9569, 1996.

Wu, H. H. and A. L. Broadfoot, The extreme ultraviolet albedos of the planet Mercury and of the Moon, J. Geophys. Res., 82, 759-761, 1977.

M. Taguchi (e-mail: taguchi@nipr.ac.jp), G. Funabashi, S. Watanabe, Y. Takahashi, and H. Fukunishi 